\title{
Diagnostic accuracy of adenosine deaminase for tuberculous peritonitis: a meta-analysis
}

Yong-chun Shen, Tao Wang, Lei Chen, Ting Yang, Chun Wan, Qian-jing Hu, Fu-qiang Wen

Division of Medicine, West China Hospital of Sichuan University, Chengdu, P.R. China

Submitted: 6 November 2012

Accepted: 21 January 2013

Arch Med Sci 2013; 9, 4: 601-607

DOI: 10.5114 /aoms.2013.36904

Copyright $\odot 2013$ Termedia \& Banach

\section{Abstract}

Introduction: Tuberculous peritonitis remains a diagnostic challenge for clinicians. Many studies have investigated the usefulness of adenosine deaminase (ADA) in ascites for the diagnosis of tuberculous peritonitis; however, the overall diagnostic accuracy of ADA for tuberculous peritonitis remains unclear. The aim of the present meta-analysis was to determine the overall accuracy of ADA measurements in the diagnosis of tuberculous peritonitis.

Material and methods: We performed a systematic search in PubMed and Embase to identify published studies that evaluated the diagnostic role of ADA for tuberculous peritonitis. Quality was assessed according to standardized Quality Assessment of Diagnostic Accuracy Studies criteria. Sensitivity, specificity and other measures of accuracy of ADA assay in order to diagnose tuberculous peritonitis were pooled using random effects models. Summary receiver operating characteristic curve (SROC) was used to summarize overall test performance.

Results: Sixteen studies met inclusion criteria for the present meta-analysis. The pooled sensitivity and specificity for diagnosing tuberculous peritonitis were 0.93 (95\% Cl: 0.89-0.95) and 0.96 (95\% Cl: 0.94-0.97), respectively. The positive likelihood ratio was 15.80 (95\% Cl: 10.87-22.95), negative likelihood ratio was 0.09 (95\% Cl: $0.05-0.16)$ and diagnostic odds ratio was $249.28(95 \% \mathrm{Cl}$ : 113.11-549.39). The area under the SROC was 0.98 .

Conclusions: Ascitic ADA determination is a relatively sensitive and specific test for the diagnosis of tuberculous peritonitis. Measurement of ADA in ascites is thus likely to be a useful diagnostic method for tuberculous peritonitis.

Key words: tuberculous peritonitis, adenosine deaminase, meta-analysis.

\section{Introduction}

Tuberculosis (TB) remains a public health challenge worldwide [1, 2]. From the latest 2012 global tuberculosis report, in 2011, it is estimated that there were 8.7 million new cases of TB and 1.4 million people died from TB, including almost one million deaths among HIV-negative individuals and 430,000 among people who were HIV-positive [1]. Tuberculous peritonitis (TBP) is one of the most frequent extra-pulmonary locations of TB, and its mortality rate may exceed $50 \%$ without timely treatment. The delayed diagnosis of TBP has been proven as the most important factor for its high mortality $[3,4]$. However, current diagnostic tests for TBP are difficult and time-consuming. The results of mycobacterial cultures might take more than 4 weeks and their sensitivity ranges from $43 \%$ to $83 \%$. In addition, the result depends on the quality of samples cultured and meth-
Corresponding author: Fu-qiang Wen MD, PhD Division of Medicine West China Hospital of Sichuan University Chengdu 610041, P.R. China Phone: 862885422380 Fax: 862885582944 E-mail: wenfuqiang.scu@gmail.com 
ods utilized, and acid-fast stained smears are disappointingly insensitive $[5,6]$. Caseous granulomas of peritoneal biopsies obtained by invasive laparoscopy or laparotomy are helpful for rapid primitive diagnosis, but the procedures may not be available in all level hospitals and well tolerated, and they may increase rates of morbidity and mortality $[7,8]$. The high mortality rate in untreated patients warrants a quick and noninvasive test for screening TBP.

Adenosine deaminase (ADA) is a purine-degrading enzyme that catalyzes the deamination of adenosine in an irreversible manner, which results in the production of inosine. Adenosine deaminase levels in body fluids can be measured rapidly, and they might provide an alternative for the diagnosis of TB [9]. Several studies reported the use of ADA in the diagnosis of TB in other fluids including meningeal, pleural, and pericardial effusions, suggesting that increasing ADA activity relates to the intensity of stimulation and the maturation state of the lymphocyte, due to the immune cellular response against Mycobacterium tuberculosis [10-12].

In fact, quite a lot of studies have investigated the diagnostic role of ascitic ADA for TBP. Considering the controversy about the current role of ADA as a diagnostic tool for TBP, the present meta-analysis aims to determine the overall diagnostic accuracy of ADA for TBP.

\section{Material and methods}

The present meta-analysis was performed according to the guidelines of the preferred reporting items for systematic reviews and meta-analysis (PRISMA) statement and with methods recommended by the Cochrane Diagnostic Test Accuracy Working Group [13, 14].

\section{Study identification}

To identify studies that evaluated the diagnostic accuracy of ADA for TBP, two independent reviewers performed a search of PubMed (Medline) and Embase up to October, 2012. The search key words included "ADA or adenosine deaminase", "tuberculosis or tuberculous", and "peritonitis". In addition, we obtained additional articles by citation tracking of review articles and original articles.

\section{Study selection}

We set the inclusion criteria as follows: measurement of ascitic ADA in human subjects; detailed diagnostic criteria for TBP; studies provided both the sensitivity and specificity of ADA assay; at least 20 participants (10 patients and 10 controls).

Exclusion criteria: no control group; limited participants; non-English publications; publications with limited information to calculate sensitivity and specificity of ADA.

The articles that were finally included in the meta-analysis were reviewed independently by two different reviewers and discrepancies in the interpretation were resolved by consensus.

\section{Data extraction}

The final set of articles was assessed independently by two reviewers, who were blinded to the article details, and the differences between them were solved by consensus. The following data from each publication were retrieved: author, publication year, participants, gold standard for TBP diagnosis, ADA assay method, sensitivity and specificity data, methodological quality, study design. If no data on the above information were presented in the primary studies, we marked it with "Not Available, NA".

\section{Assessment of study quality}

To assess trial methodology, included publications were reviewed independently by two authors and given a quality score by using the QUADAS (quality assessment for studies of diagnostic accuracy, an evidence-based quality assessment tool to be used in systematic reviews of diagnostic accuracy studies, maximum score 14 ) tools [15].

\section{Data synthesis and statistical analysis}

The standard methods recommended for diagnostic accuracy meta-analyses were used in the present study [16]. The following indexes of test accuracy were computed for each study: sensitivity, specificity, positive likelihood ratio (PLR), negative likelihood ratio (NLR), and diagnostic odds ratio (DOR). The analysis was based on a summary receiver operating characteristic (SROC) curve [17]. Heterogeneity was evaluated by using the $\chi^{2}$ test and $l^{2}$ test. The random effects model was performed to synthesize data when heterogeneity was present $(p<0.05$ and $R>50 \%$ ); otherwise the fixed effects model was used. Since publication bias is of concern for meta-analyses of diagnostic studies, we tested for the potential presence of this bias using Deeks' funnel plots [18].

All analyses were performed using two statistical software programs (Meta-DiSc for Windows; XI Cochrane Colloquium, Barcelona, Spain and Stata, version 12; Stata Corporation, College Station, TX, USA). All statistical tests were two-sided, and significance was set at $p<0.05$.

\section{Results}

After independent review, sixteen studies in fifteen publications with 1574 subjects on the use of ADA in patients with ascites were considered eligi- 
ble for inclusion in the present meta-analysis [1933]. The major reasons for excluding other studies were as follows: non-diagnostic studies or studies cannot reconstruct the diagnostic 2 by 2 table; limited samples, or mixed with other serous effusions.

\section{Study characteristics and quality report}

Of the included studies, the average sample size in the 16 studies was 49 (41-368). For most studies, the diagnosis of TBP was based on bacteriological or histological examinations or both, four studies included some patients who were diagnosed with TBP based on clinical diagnosis, including clinical presentation, pleural fluid analysis, radiology and the responsiveness to anti-tuberculosis chemotherapy [20, 22, 29, 32]. For ADA assay method, the Giusti method was applied in 11 studies and non-Giusti methods were used in 5 studies. The cut-off value was $\geq 30 \mathrm{IU} / \mathrm{l}$ in 14 studies (30-40 IU/I), and< $30 \mathrm{IU} / \mathrm{I}$ in 2 studies; one was 21 IU/I [19], and the other one was 7 IU/I [26]. The quality of the sixteen studies was generally high with ten studies having QUADAS scores $\geq 10$. The clinical summary of these studies, along with the QUADAS scores, are outlined in Table I.

\section{Diagnostic accuracy for TBP}

Heterogeneity examination is performed to choose the appropriate calculation model; the heterogeneity analysis showed $R$ of $54.1 \%$ for sensitivity and $59.2 \%$ for specificity, suggesting significant heterogeneity among included studies; thus the random effects model approach was selected for the present meta-analysis. The forest plots of the sensitivity and specificity for ADA assays in diagnosing TBP are shown in Figures 1 and 2, respectively. The pooled sensitivity was $0.93(95 \% \mathrm{Cl}$ : 0.89-0.95), specificity was 0.96 (95\% Cl: 0.94-0.97). The PLR was 15.80 (95\% Cl: 10.87-22.95), the NLR was 0.09 (95\% Cl: $0.05-0.16)$ and the DOR was 249.28 (95\% Cl: 113.11-549.39).

Figure 3 shows the SROC plotting the true-positive against the false-positive rates of individual studies. The area under the curve (AUC) was 0.98, indicating that the level of overall accuracy was high.

We conducted a sub-group analysis, for the 11 studies that determined ascitic ADA with the Giusti method. The pooled sensitivity was 0.96 (95\% Cl: 0.93-0.98), specificity was 0.96 (95\% Cl: 0.94-0.97), PLR was 13.82 (95\% Cl: 12.58-26.68), NLR was 0.07 (95\% Cl: 0.04-0.11) and the DOR was 509.44 (95\% Cl: 227.66-1139.95). The AUC was 0.99. Thus, the Giusti method may be a suitable method for determination of ascitic ADA.

\section{Publication bias}

Deeks' funnel plot asymmetry test was used to evaluate potential publication bias. The statistical- ly non-significant value $(p=0.86)$ for the slope coefficient suggests symmetry in the data and a low likelihood of publication bias (Figure 4).

\section{Discussion}

Tuberculous peritonitis is still a public health problem in endemic regions of the world. It may be fatal but is medically cured if diagnosed in a timely fashion. To make an early and accurate diagnosis is of great importance for its prognosis. Adenosine deaminase is a well-known diagnostic marker for tuberculosis. In fact, Riquelme et al. conducted a meta-analysis to analyze the diagnostic role ADA for TBP. According to his inclusion criteria, only four publications were included [34]. Several years have passed, and some new studies have been added, so we conducted this updated meta-analysis. According to our inclusion criteria, we included the most recent published studies, and included studies using a non-Giusti method. The number of included studies provides enough evidence to support the diagnostic power of ADA for TBP. We found a summary AUC of 0.98 , a summary estimate of 0.93 for sensitivity and 0.96 for specificity. It seems that ADA assay plays a valuable role in the diagnosis of TBP. Both Riquelme's and our studies support the proposition that ADA determination is a discriminating test for diagnosing TBP [34].

The SROC curve has been recommended to represent the overall performance of a diagnostic study, which shows the trade-off between sensitivity and specificity, based on data from a metaanalysis [35]. The AUC and index $Q\left(^{*}\right)$ are recognized as potentially useful summaries of the curve. Q-value, the intersection point of the SROC curve with a diagonal line from the left upper corner to the right lower corner of the ROC space, which corresponds to the highest common value of sensitivity and specificity for the test, represents an overall measure of the discriminatory power of a test. In the present study, the Q value was 0.94 , and the maximum joint sensitivity and specificity of ADA for TBP was 0.94. The AUC also represents the overall accuracy of the diagnostic study. The AUC represents an analytical summary of test performance and displays the trade-off between specificity and sensitivity. If the AUC is 1 , it means the ADA test differentiates perfectly between TBP and non-TBP patients. An AUC of greater than 0.9 indicates high diagnostic accuracy. In the present meta-analysis, the AUC was 0.98, suggesting that the level of overall accuracy of ascitic ADA for TBP is high.

Apart from SROC, we also examined other diagnostic indexes. DOR is a single indicator of diagnostic accuracy that combines the data from sensitivity and specificity into a single number [36]. It is defined as the ratio of the odds of positive test results in the diseased relative to the odds of pos- 

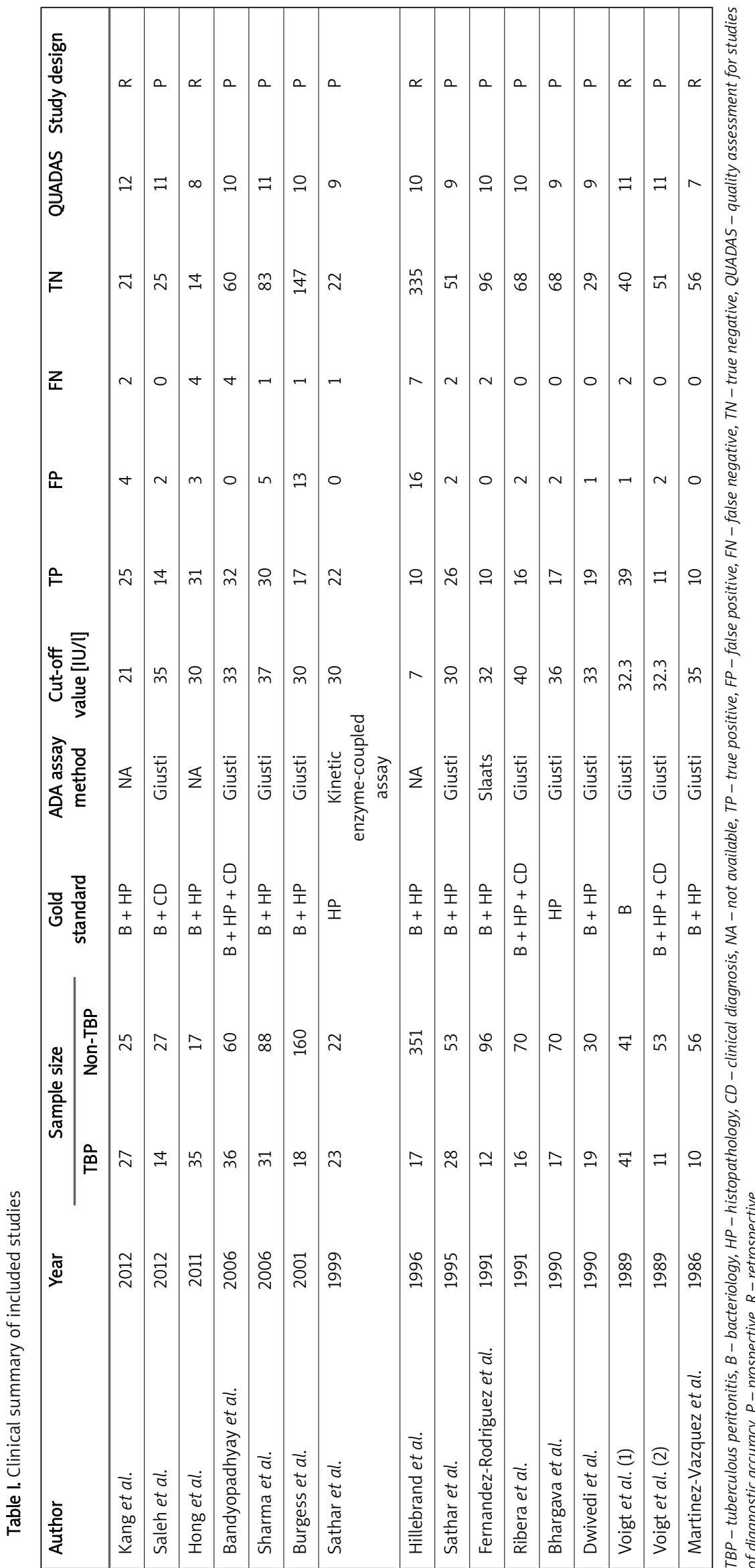


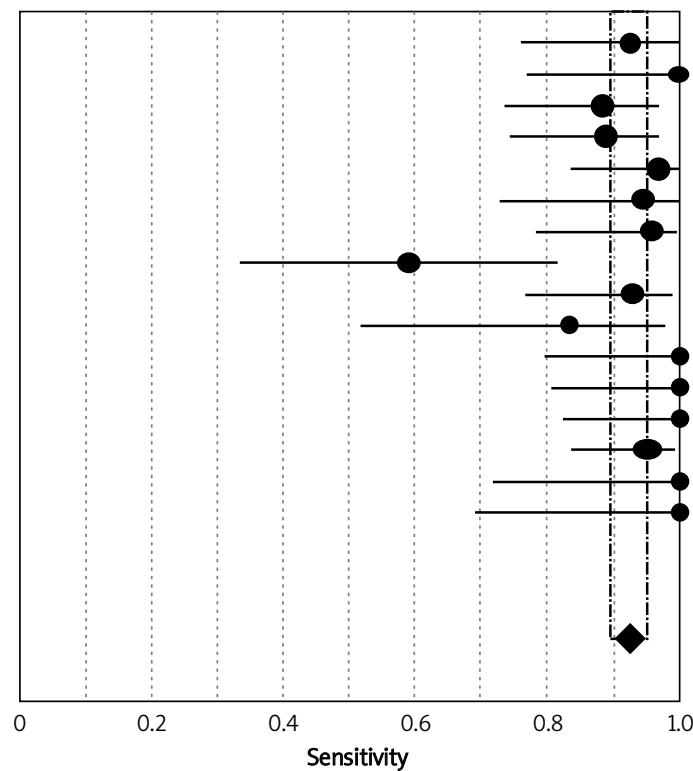

Kang et al.

Saleh et al.

Hong et al.

Bandyopadhyay et al.

Sharma et al.

Burgess et al.

Sathar et al.

Hillebrand et al.

Sathar et al.

Fernandez-Rodriguez et al.

Ribera et al.

Bhargava et al.

Dwivedi et al.

Voigt et al. (1)

Voigt et al. (2)

Martinez-Vazquez et al.
Sensitivity $(95 \% \mathrm{Cl})$

$0.93(0.76-0.99)$

$1.00(0.77-1.00)$

$0.89(0.73-0.97)$

$0.89(0.74-0.97)$

097 (0.83-1.00)

$0.94(0.73-1.00)$

$0.96(0.78-1.00)$

$0.59(0.33-0.82)$

$0.93(0.76-0.99)$

$0.83(0.52-0.98)$

$1.00(0.79-1.00)$

$1.00(0.80-1.00)$

$1.00(0.82-1.00)$

$0.95(0.83-0.99)$

$1.00(0.72-1.00)$

$1.00(0.69-1.00)$

Figure 1. Forest plots of pooled sensitivity of ADA for the diagnosis of TBP. The point estimates of sensitivity from each study are shown as solid circles. Error bars indicate $95 \%$ confidence intervals

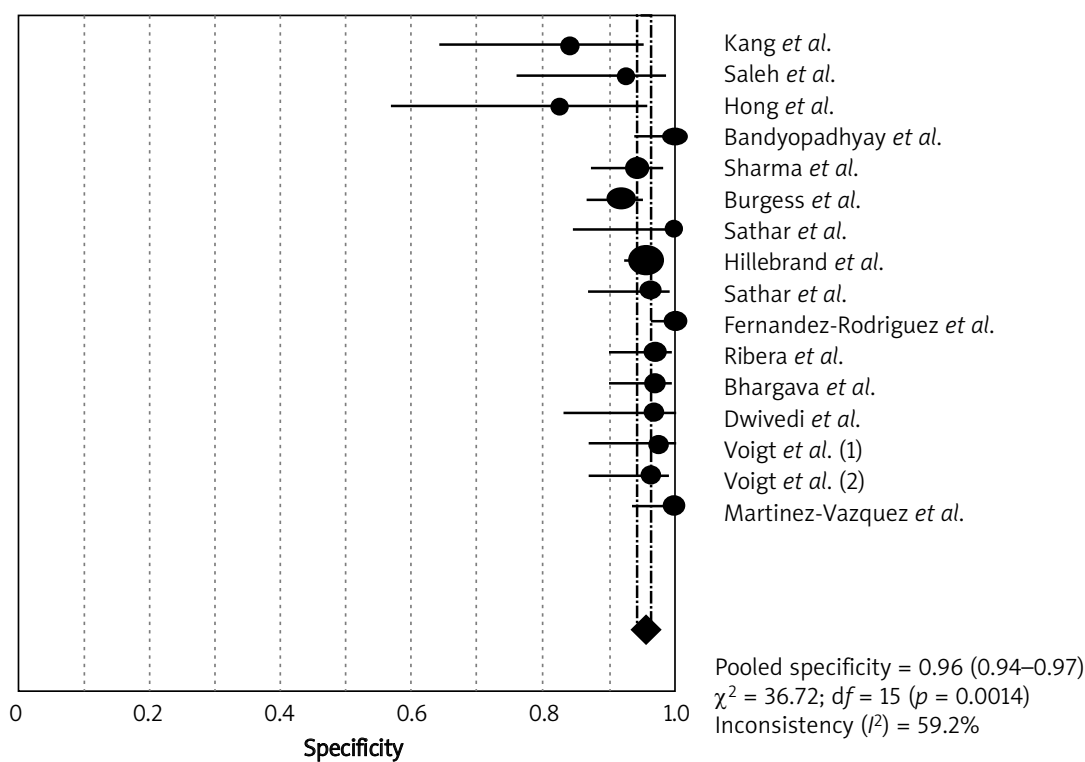

Specificity $(95 \% \mathrm{Cl})$

$0.843(0.64-0.95)$

$0.93(0.76-0.99)$

$0.82(0.57-0.96)$

$1.00(0.94-1.00)$

$0.94(0.87-0.98)$

$0.92(0.87-0.96)$

$1.00(0.85-1.00)$

$0.95(0.93-0.97)$

$0.96(0.87-1.00)$

$1.00(0.96-1.00)$

$0.97(0.90-1.00)$

$0.97(0.90-1.00)$

$0.97(0.83-1.00)$

$0.98(0.87-1.00)$

$0.96(0.87-1.00)$

$1.00(0.94-1.00)$

Figure 2. Forest plots of pooled specificity of ADA for the diagnosis of TBP. The point estimates of specificity from each study are shown as solid circles. Error bars indicate $95 \%$ confidence intervals

itive test results in the non-diseased. The value of DOR ranges from 0 to infinity, with higher values indicating better discriminatory test performance. In the present meta-analysis, the pooled DOR was 249.28, suggesting that ADA assays seemed to be useful in the diagnosis of TBP. Since the SROC curve and DOR are not easy to interpret and use in clinical practice, likelihood ratios are considered more clinically meaningful [37]. The PLR was 15.80 , indicating that patients with TBP have about 16-fold higher chance of being ADA assay-positive compared with non-TBP subjects. The NLR was 0.09; it means that if the ADA assay result was negative, the probability that this subject has TBP is only $9 \%$, which is low enough to exclude TBP.

Our meta-analysis suggests that ADA determination plays a valuable role in diagnosing TBP. The reported sensitivities varied among studies; only two studies had sensitivity less than 0.85 , and the pooled sensitivity was 0.93 . Ascitic ADA assay is suitable as a routine screening tool for TBP. Physicians may argue that ADA assay results may be affected by liver cirrhosis. Liao et al. confirmed that even with lower ascites ADA activity in patients 


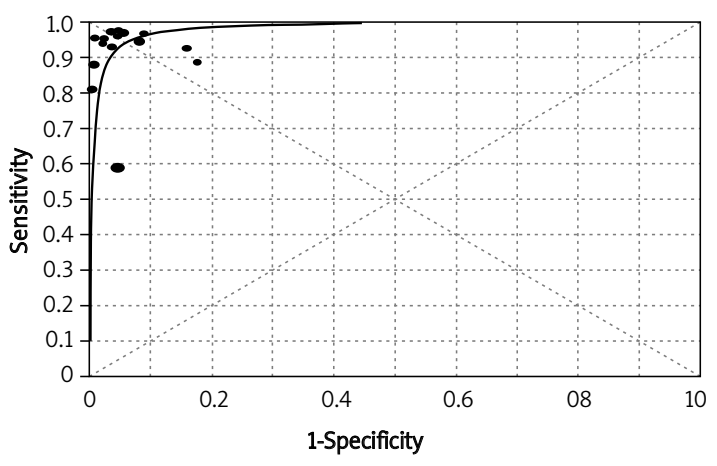

Figure 3. Summary receiver operating characteristic (SROC) curve of ADA for the diagnosis of TBP. The size of each solid circle represents the size of each study included in the present meta-analysis. The regression SROC curve indicates the overall diagnostic accuracy

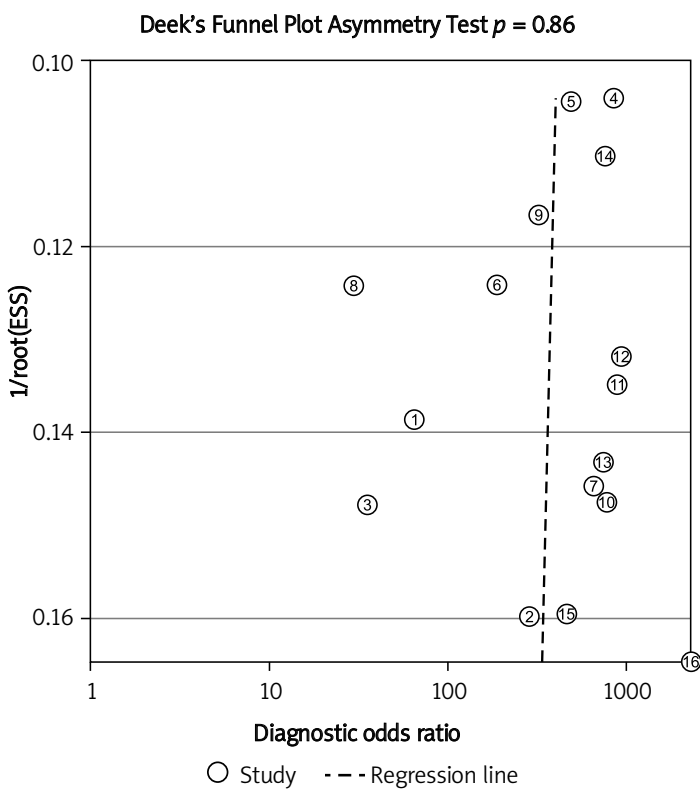

Figure 4. Linear regression test of funnel plot asymmetry. The statistically non-significant value $(p=0.86)$ for the slope coefficient suggests symmetry in the data and a low likelihood of publication bias

with liver cirrhosis, ascites ADA levels could be significantly elevated resulting from strong immune responses when cirrhotic patients suffer from TBP [38]. Considering its high sensitivity and specificity, ascitic ADA may be useful in the differential diagnosis of TBP. In patients with underlying cirrhosis, concomitant cirrhosis should not limit its clinical utility [38]. Although peritoneal biopsies obtained by laparoscopy or laparotomy are valuable for rapid diagnosis of TBP, these invasive procedures may not be available in all hospitals and increase mortality $[7,8]$. Thus, the importance of the ADA test is that it not only provides high diagnostic accuracy, but also guides the inclusion of patients who might benefit from further invasive procedures.

Based on the evidence compiled in this metaanalysis, ascitic ADA measurement plays a critical role in the diagnosis of TBP. It is likely to be a useful diagnostic tool for TBP. In addition, the results of ascitic ADA assays should be interpreted in parallel with clinical findings and the results of traditional tests such as microbiologic examination and peritoneal biopsy. It should be noted that there are currently no ascitic markers (including ADA) which are specific for TBP. Further studies aim to investigate the diagnostic performance of other ascitic markers, such as interferon- $\gamma$ [23], or combined diagnostic accuracy of different ascitic markers should be performed.

\section{Acknowledgments}

This work is supported by grants 81230001 and 31171103 from the National Natural Science Foundation of China and grants 00-722 and 06-834 from the China Medical Board of New York to Dr. Fuqiang Wen. Yongchun Shen and Tao Wang contributed equally to this work. In addition, we are indebted to the authors of the primary studies; without their contributions, this work would have been impossible.

\section{References}

1. World Health Organization. Global tuberculosis report 2012. World Health Organization 2012.

2. Kiran B, Cagatay T, Clark P. Can immune parameters be used as predictors to distinguish between pulmonary multidrug-resistant and drug-sensitive tuberculosis? Arch Med Sci 2010; 6: 77-82.

3. Chow KM, Chow VC, Hung LC, Wong SM, Szeto CC. Tuberculous peritonitis-associated mortality is high among patients waiting for the results of mycobacterial cultures of ascitic fluid samples. Clin Infect Dis 2002; 35: 409-13.

4. Ahmad M, Ahmed A. Tuberculous peritonitis: fatality associated with delayed diagnosis. South Med J 1999; 92: 406-8.

5. Inadomi JM, Kapur S, Kinkhabwala M, et al. The laparoscopic evaluation of ascites. Gastrointest Endosc Clin N Am 2001; 11: 79-91.

6. Shakil AO, Korula J, Kanel GC, Murray NG, Reynolds TB. Diagnostic features of tuberculous peritonitis in the absence and presence of chronic liver disease: a case control study. Am J Med 1996; 100: 179-85.

7. Guirat A, Koubaa M, Mzali R, et al. Peritoneal tuberculosis. Clin Res Hepatol Gastroenterol 2011; 35: 60-9.

8. Samples J, Meyers MO. Diagnostic difficulties associated with peritoneal tuberculosis. Am Surg 2012; 78: E381-2.

9. Giusti, G. Adenosine deaminase. In: Methods of enzymatic analysis. Bergmeyer HU (ed.). Academic Press, New York 1974; 1092-9.

10. Tuon FF, Higashino HR, Lopes MI, et al. Adenosine deaminase and tuberculous meningitis: a systematic review with meta-analysis. Scand J Infect Dis 2010; 42: 198-207.

11. Liang QL, Shi HZ, Wang K, et al. Diagnostic accuracy of adenosine deaminase in tuberculous pleurisy: a metaanalysis. Respir Med 2008; 102: 744-54.

12. Tuon FF, Litvoc MN, Lopes MI. Adenosine deaminase and tuberculous pericarditis; a systematic review with metaanalysis. Acta Trop 2006; 99: 67-74.

13. Moher D, Liberati A, Tetzlaff J, et al. Preferred reporting items for systematic reviews and meta-analyses: the PRISMA statement. PLoS Med 2009; 6: e1000097. 
14. Leeflang MM, Deeks JJ, Gatsonis C, et al. Systematic reviews of diagnostic test accuracy. Ann Intern Med 2008; 149: 889-97.

15. Whiting P, Rutjes AW, Reitsma JB, et al. The development of QUADAS: a tool for the quality assessment of studies of diagnostic accuracy included in systematic reviews. BMC Med Res Methodol 2003; 3: 25.

16. Devillé WL, Buntinx F, Bouter LM, et al. Conducting systematic reviews of diagnostic studies: didactic guidelines. BMC Med Res Methodol 2002; 2: 9.

17. Walter SD. Properties of the summary receiver operating characteristic (SROC) curve for diagnostic test data. Stat Med 2002; 21: 1237-56.

18. Deeks JJ, Macaskill P, Irwig L. The performance of tests of publication bias and other sample size effects in systematic reviews of diagnostic test accuracy was assessed. J Clin Epidemiol 2005; 58: 882-93.

19. Kang SJ, Kim JW, Baek JH, et al. Role of ascites adenosine deaminase in differentiating between tuberculous peritonitis and peritoneal carcinomatosis. World J Gastroenterol 2012; 18: 2837-43.

20. Saleh MA, Hammad E, Ramadan MM, et al. Use of adenosine deaminase measurements and QuantiFERON in the rapid diagnosis of tuberculous peritonitis. J Med Microbiol 2012; 61: 514-9.

21. Hong KD, Lee SI, Moon HY. Comparison between laparoscopy and noninvasive tests for the diagnosis of tuberculous peritonitis. World J Surg 2011; 35: 2369-75.

22. Bandyopadhyay R, Bandyopadhyay SK, Ghosal J, et al. Study of biochemical parameters of ascitic fluid in exudative ascites with special reference to tuberculous peritonitis. J Indian Med Assoc 2006; 104: 174, 176-7, 185.

23. Sharma SK, Tahir M, Mohan A, et al. Diagnostic accuracy of ascitic fluid IFN-gamma and adenosine deaminase assays in the diagnosis of tuberculous ascites. J Interferon Cytokine Res 2006; 26: 484-8.

24. Burgess LJ, Swanepoel CG, Taljaard JJ. The use of adenosine deaminase as a diagnostic tool for peritoneal tuberculosis. Tuberculosis (Edinb) 2001; 81: 243-8.

25. Sathar MA, Ungerer JP, Lockhat F, et al. Elevated adenosine deaminase activity in patients with HIV and tuberculous peritonitis. Eur J Gastroenterol Hepatol 1999; 11: 337-41.

26. Hillebrand DJ, Runyon BA, Yasmineh WG, et al. Ascitic fluid adenosine deaminase insensitivity in detecting tuberculous peritonitis in the United States. Hepatology 1996; 24: 1408-12.

27. Sathar MA, Simjee AE, Coovadia YM, et al. Ascitic fluid gamma interferon concentrations and adenosine deaminase activity in tuberculous peritonitis. Gut 1995; 36 : 419-21.

28. Fernandez-Rodriguez CM, Perez-Arguelles BS, Ledo L, et al. Ascites adenosine deaminase activity is decreased in tuberculous ascites with low protein content. Am J Gastroenterol 1991; 86: 1500-3.

29. Ribera E, Martínez Vásquez JM, Ocaña I, et al. Diagnostic value of ascites gamma interferon levels in tuberculous peritonitis. Comparison with adenosine deaminase activity. Tubercle 1991; 72: 193-7.

30. Bhargava DK, Gupta M, Nijhawan S, et al. Adenosine deaminase (ADA) in peritoneal tuberculosis: diagnostic value in ascitic fluid and serum. Tubercle 1990; 71: 121-6.

31. Dwivedi M, Misra SP, Misra V, et al. Value of adenosine deaminase estimation in the diagnosis of tuberculous ascites. Am J Gastroenterol 1990; 85: 1123-5.

32. Voigt MD, Kalvaria I, Trey C, et al. Diagnostic value of ascites adenosine deaminase in tuberculous peritonitis. Lancet 1989; 1: 751-4.
33. Martinez-Vazquez JM, Ocaña I, Ribera E, et al. Adenosine deaminase activity in the diagnosis of tuberculous peritonitis. Gut 1986; 27: 1049-53.

34. Riquelme A, Calvo M, Salech F, et al. Value of adenosine deaminase (ADA) in ascitic fluid for the diagnosis of tuberculous peritonitis: a meta-analysis. J Clin Gastroenterol 2006; 40: 705-10.

35. Jones CM, Athanasiou T. Summary receiver operating characteristic curve analysis techniques in the evaluation of diagnostic tests. Ann Thorac Surg 2005; 79: 16-20.

36. Glas AS, Lijmer JG, Prins MH, et al. The diagnostic odds ratio: a single indicator of test performance. J Clin Epidemiol 2003; 56: 1129-35.

37. Crewe $S$, Rowe PC. Research and statistics: likelihood ratio in diagnosis. Pediatr Rev 2011; 32: 296-8.

38. Liao YJ, Wu CY, Lee SW, et al. Adenosine deaminase activity in tuberculous peritonitis among patients with underlying liver cirrhosis. World J Gastroenterol 2012; 18: 5260-5. 